\title{
Natural Convection in Porous Triangular Enclosure with a Circular Obstacle in Presence of Heat Generation
}

\author{
Raju Chowdhury $^{1,2}$, Md. Abdul Hakim Khan ${ }^{2}$, Md. Noor-A-Alam Siddiki ${ }^{1}$ \\ ${ }^{1}$ Department of Natural Science, Stamford University Bangladesh, Dhaka-1209, Bangladesh \\ ${ }^{2}$ Department of Mathematics, Bangladesh University of Engineering \& Technology, Dhaka-1000, Bangladesh
}

Email address:

rajumath@stamforduniversity.edu.bd (R. Chowdhury)

\section{To cite this article:}

Raju Chowdhury, Md. Abdul Hakim Khan, Md. Noor-A-Alam Siddiki. Natural Convection in Porous Triangular Enclosure with a Circular Obstacle in Presence of Heat Generation. American Journal of Applied Mathematics. Vol. 3, No. 2, 2015, pp. 51-58.

doi: $10.11648 /$ j.ajam.20150302.14

\begin{abstract}
The flow and heat transfer due to natural convection in a triangular enclosure filled with a fluid saturated porous medium with a circular body in presence of heat generation has been numerically analyzed. The bottom wall of the enclosure is heated at a constant temperature while the left and right inclined wall of the cavity is maintained at cold temperature. The cavity contains a circular body which is insulated. The governing equations are solved numerically subject to suitable boundary conditions by Galerkin's weighted residuals scheme of finite element method. Results are presented by streamlines, isotherms, mean Nusselt numbers for the variant parameters such as heat generation $(\lambda)$ and radius of the circular body $(R)$. Prandtl number $(P r)$ and Rayleigh number $(R a)$ are considered unchanged. It is found that these parameters have significant effect on the flow and temperature fields inside the cavity.
\end{abstract}

Keywords: Natural Convection, Heat Generation, Triangular Cavity, Circular Body, Porous Media

\section{Introduction}

Free convection flow and heat transfer in porous media have been receiving a considerable attention in the literature. This attention stems from its importance in many applications of engineering such as building insulation, grain storage, geothermal problems and solar collectors. These applications are well described by Nield and Bejan [1] and Ingham and Pop [2].

Most of the researchers have chosen rectangular geometries [3-5] in porous media due to simplicity of solutions and practical importance. Some researchers have investigated non-rectangular geometric shaped enclosures such as triangular [6-8], trapezoidal [9], and wavy shapes [10]. In these studies they have shown that both flow and temperature fields are affected by the geometrical parameters.

Triangular cavities with differentially heated isothermal wall have received a considerable attention because of its wide applications for roof-building, design of industrial equipments, cooling of electronic devices, furnaces and fire control in building and some solar applications.

Recent studies on natural convection in triangular shaped cavities have also been carried out for various applications.
Varol et al. [11], Ridouane et al. [12], Joudi et al. [13] studied numerically, the performance of a prism shaped solar collector with a right angled triangular cross-sectional area. The effect of horizontal adiabatic partition has been studied. Tzeng et al. [14] carried out numerical simulation and parametric studies for triangular enclosures. The aim of this study is to establish efficient energy management.

The phenomenon of heat and fluid flow for a configuration of isothermal vertical walls, controlled at different temperatures and with adiabatic horizontal walls are well explained [3]. The study of natural convection heat transfer in a triangular enclosure filled with a porous medium has been first considered by Bejan and Poulikakos [15]. They investigated that the porous media can be a control element for heat transfer and fluid flow. Varol et al. [16] have studied natural convection in a right angle triangular enclosure in a porous medium by taking into account all possibilities of thermal boundary conditions. Basak et al. [17] have made a numerical analyzes using finite element method to investigate the effects of non-isothermal boundary conditions on flow fields and temperature distribution in a triangular enclosure filled with a porous medium.

Many authors have recently examined heat transfer in cavities with partitions, fins and block which controls the 
convective flow phenomenon. A body can be used as a control element for heat transfer and fluid flow which is analyzed by Varol et al. [18]. Amine et al. [19] investigated the thermal convection around obstacles with different configurations. Ha et al. [20] tested the different boundary conditions for the inserted body to the enclosure. They found that the presence of the body obstructs the flow and temperature fields. Oztop et al. [21] analyzed the effects of the location of the insulated body for partially heated enclosure.

To the best of the authors' knowledge, no attention has been paid to the problem of natural convection of flow and heat transfer in an equilateral triangular enclosure containing an insulated circular body and heat generation. The objective of the present study is to examine the effect on size of the circular body on free convection heat transfer in porous medium filled equilateral triangular enclosure with heat generation. The enclosure is constantly heated from bottom wall and cold temperature is maintained at left and right inclined wall. The tests were performed for different heat generation parameter and radius of the circular body.

\section{Physical Formulation}

The treated problem of the two-dimensional porous triangular enclosure of height $L$ is shown in Fig. 1(a) with the corresponding boundary conditions. A insulated circular body of radius $r$ is inserted to the center of the enclosure. The bottom wall is heated isothermally with temperature $T_{h}$. The left and right inclined wall is cold and maintained at temperature $T_{c}$ such that $T_{c}<T_{h}$. However, gravitional force acts in vertical direction.

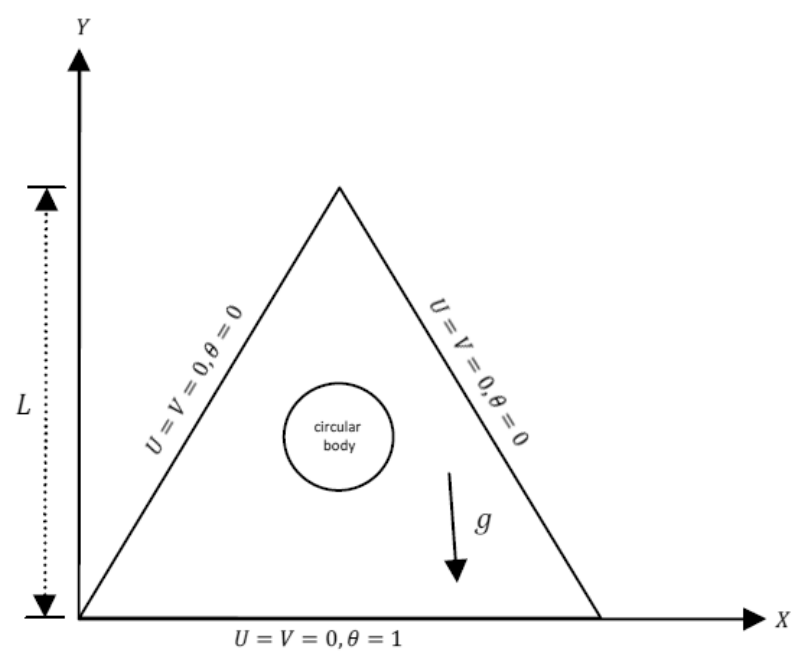

Fig. 1. Schematic diagram of the enclosure with boundary conditions

\section{Mathematical Formulation}

In this study, we assume steady, two-dimensional, laminar flow of a viscous incompressible fluid having constant properties. Under Boussinesq approximation the dimensionless equations describing the flow are as follows:

$$
\begin{gathered}
\frac{\partial U}{\partial X}+\frac{\partial V}{\partial Y}=0 \\
U \frac{\partial U}{\partial X}+V \frac{\partial U}{\partial Y}=-\frac{\partial P}{\partial X}+\operatorname{Pr}\left(\frac{\partial^{2} U}{\partial X^{2}}+\frac{\partial^{2} U}{\partial Y^{2}}\right)-\frac{P r}{D a} U \\
U \frac{\partial V}{\partial X}+V \frac{\partial V}{\partial Y}=-\frac{\partial P}{\partial Y}+\operatorname{Pr}\left(\frac{\partial^{2} V}{\partial X^{2}}+\frac{\partial^{2} V}{\partial Y^{2}}\right)-\frac{P r}{D a} V+\operatorname{RaPr} \theta \\
U \frac{\partial \theta}{\partial X}+V \frac{\partial \theta}{\partial Y}=\left(\frac{\partial^{2} \theta}{\partial X^{2}}+\frac{\partial^{2} \theta}{\partial Y^{2}}\right)+\lambda \theta
\end{gathered}
$$

where $\operatorname{Pr}=\frac{v}{\alpha}$ is the Prandtl number, $R a=\frac{g \beta\left(T_{h}-T_{C}\right) L^{3}}{\alpha v}$ is the Rayleigh number, $D a=\frac{1}{\varepsilon}=\frac{K}{L^{2}}$ is the Darcy number, and $\lambda=\frac{Q_{0} L^{2}}{\rho v C_{p}}$ is the heat generation parameter.

The above equations are non-dimensionalized by using the following dimensionless dependent and independent variables:

$$
\begin{aligned}
& X=\frac{x}{L}, \quad Y=\frac{y}{L}, \quad P=\frac{p}{\rho U_{0}^{2}}, \quad \ell=\frac{l}{L}, \\
& R=\frac{r}{L}, \quad U=\frac{u}{U_{0}}, \quad V=\frac{v}{U_{0}}, \quad \theta=\frac{T-T_{c}}{T_{h}-T_{c}}
\end{aligned}
$$

where $U_{0}=\frac{\alpha}{L}$ is the reference velocity. where $\mathrm{X}$ and $Y$ are the coordinates varying along horizontal and vertical axes respectively, $U$ and $V$ are the velocity components along the $X$ and $Y$ directions respectively, $\theta$ is the dimensionless temperature and $P$ is the non-dimensional pressure.

In the present investigation, we have chosen $\operatorname{Pr}=0.71$, $R a=10^{5}$ and $D a=0.4$.

The dimensionless initial and boundary conditions are as follows:

All boundaries are rigid and no-slip; $U=V=0$

On the bottom wall: $\theta=1$

On the left and right inclined wall: $\theta=0$

At the circular body:

On the boundary: $U=V=0 ; \frac{\partial \theta}{\partial \mathrm{n}}=0$

Local and mean Nusselt numbers of the bottom wall are calculated via Eqn. (6) and (7) respectively,

$$
N u_{x}=\frac{h L}{k}=-\frac{\partial \theta}{\partial n} L
$$

where, $h$ represents the heat transfer coefficient, $k$ thermal conductivity and $n$ the coordinate direction normal to the surfaceand

$$
N u=\int_{0}^{b} N u_{x} d X
$$

where $b$ is the dimensionless base of the enclosure.

\section{Solution Methodology}

The numerical procedure used in this work is based on the Galerkin weighted residual method of finite element formulation. In this method, the solution domain is discretized into finite element meshes, which are composed of non-uniform triangular elements. Then the nonlinear governing partial differential equations (i.e. mass, 
momentum and energy equations) are transferred into a system of integral equations by applying Galerkin weighted residual method. The integration involved in each term of these equations is performed by using Gauss's quadrature method. The nonlinear algebraic equations so obtained are modified by imposition of boundary conditions. These modified nonlinear equations are transferred into linear algebraic equations by using Newton's method. Finally, these linear equations are solved by using Triangular Factorization method.

Table 1. Grid sensitivity check at $\operatorname{Pr}=0.71, \mathrm{Ra}=10^{5}, \mathrm{Da}=0.4, R=$ 0.01 and $\lambda=0$

\begin{tabular}{lllll}
\hline Nodes & 4382 & 7617 & 9900 & 16423 \\
(elements) & $(499)$ & $(918)$ & $(1221)$ & $(1773)$ \\
$\mathrm{Nu}$ & 7.92188 & 7.88653 & 7.91403 & 7.91403 \\
Time (s) & 106 & 196 & 213 & 321 \\
\hline
\end{tabular}

\section{Grid Sensitivity Test}

In order to determine the proper grid size for this study, a grid independence test is conducted with four types of mesh
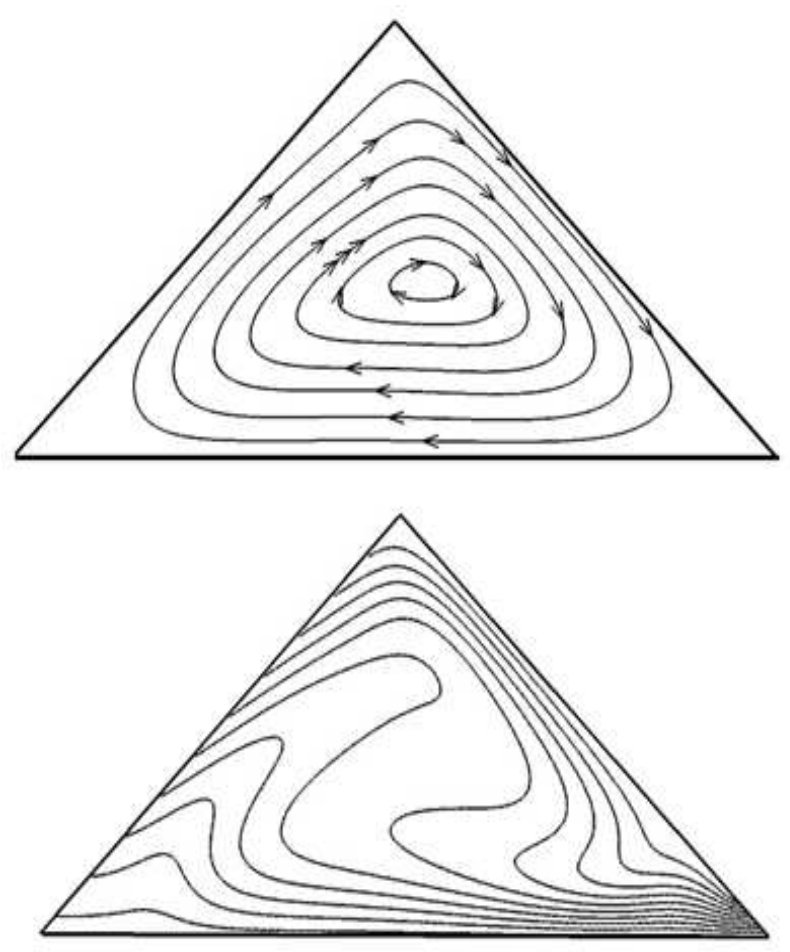

Fig. 2. A comparison for streamlines (top row) and isotherms (bottom row) between Saha and Gu [22] (on the left) and present study (on the right) for $\operatorname{Ra}=10^{5}, \operatorname{Pr}=0.7, \mathrm{Da}=0.4$ and $\lambda=0$.

\section{Result and Discussion}

The natural convection phenomenon with heat generation effect inside a equilateral triangular obstructed cavity containing a insulated circular obstacle is influenced by different controlling parameters such as $R a, P r, \lambda, D a$ and for $\quad r=0.71, R a=10^{5}, R=0.01, D a=0.4$ and $\lambda=0$ which are shown in Table 1 . The extreme values of average Nusselt number $(\mathrm{Nu})$ are used as the sensitivity measure of the accuracy of the solution and are selected as the monitoring variable. Considering both the accuracy of numerical values and computational time, the present calculations are performed with 9,900 nodes and 1,221 elements grid system.

\section{Model Validation}

The numerical simulation for pure fluid without the effect of circular obstacle has been investigated by Saha and Gu [22] for diverse values of heat generation parameter with $R a=$ $10^{5}$ and $\mathrm{Pr}=0.7$.A test has made to contrast the results obtained by the current model with earlier study [22]. Fig. 2 represents the result of streamlines and isotherms for free convection in a porous triangular cavity. The left column presents the result of Saha and $\mathrm{Gu}$ [22] and right column shows the result for current study. The comparison shows that the present's results agree with the numerical solution of Saha and Gu [22].
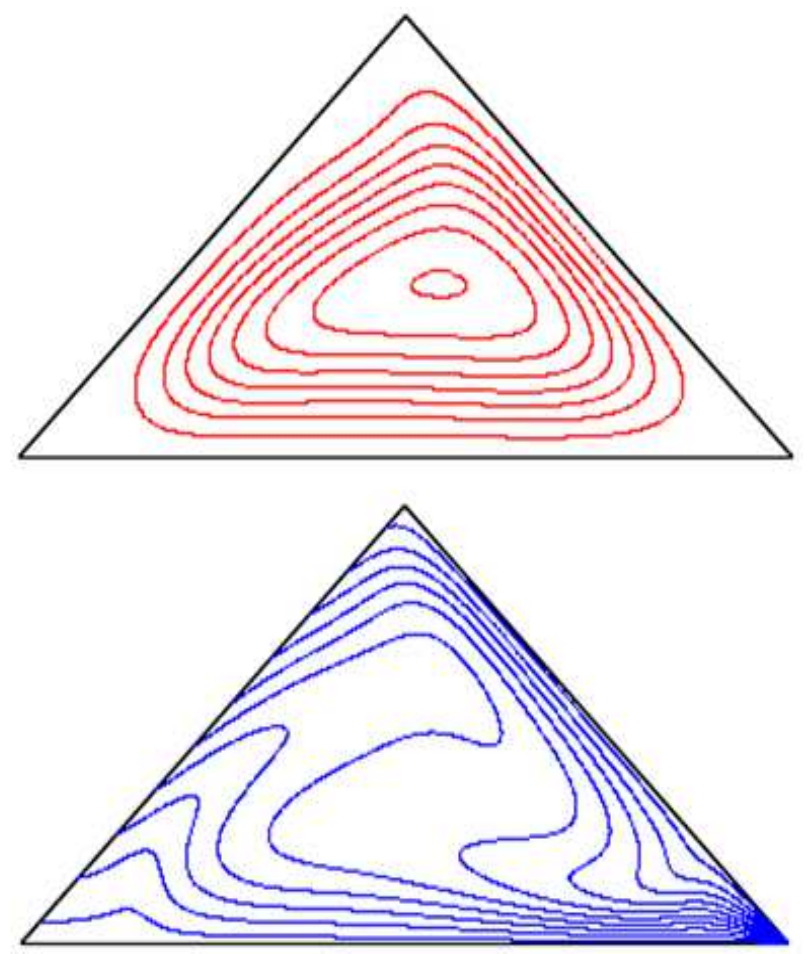

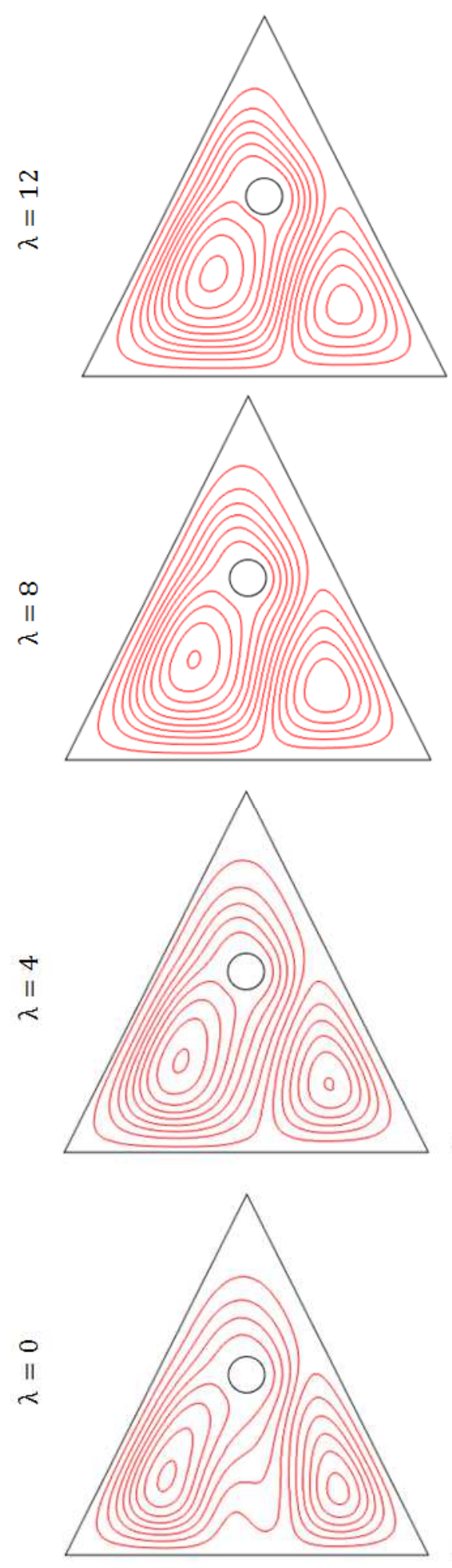

$R=0.005$
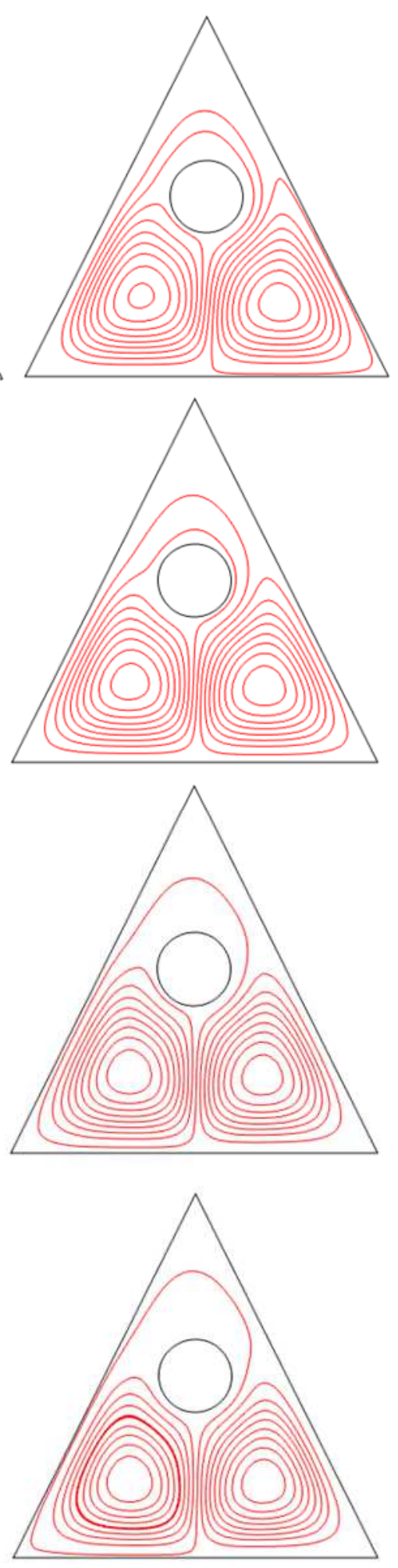

$R=0.01$
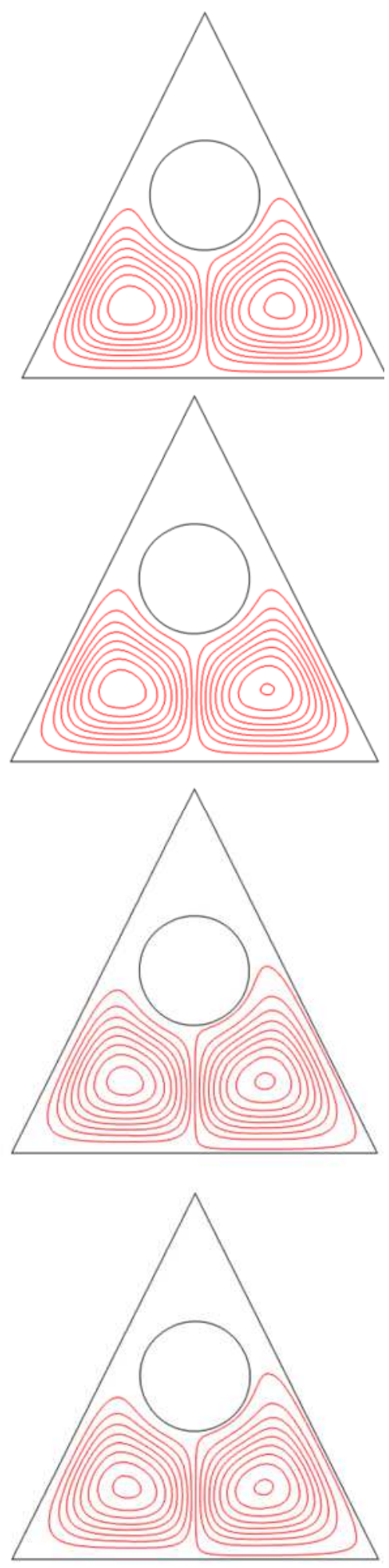

$R=0.015$

Fig. 3. Streamlines for different values of radius of the circular body $R$ and heat generation parameter $\lambda$ with $\operatorname{Ra}=10^{5}, \operatorname{Pr}=0.71$ and $D a=0.4$. 

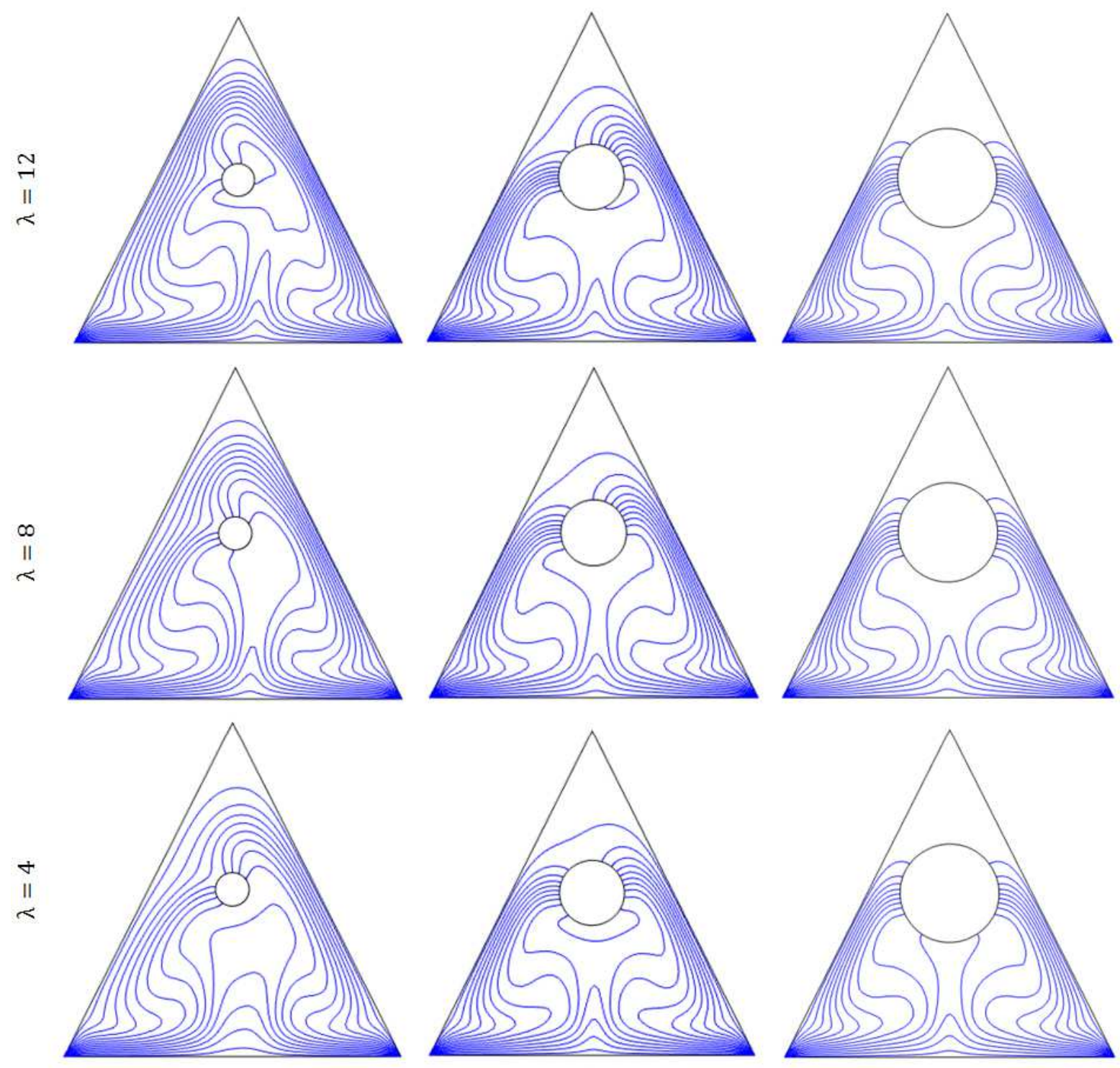

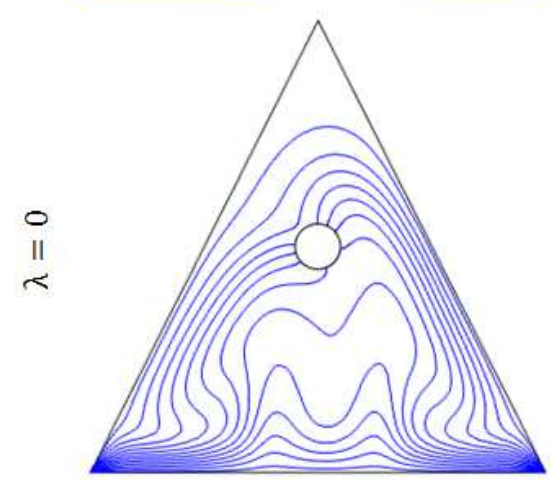

$R=0.005$

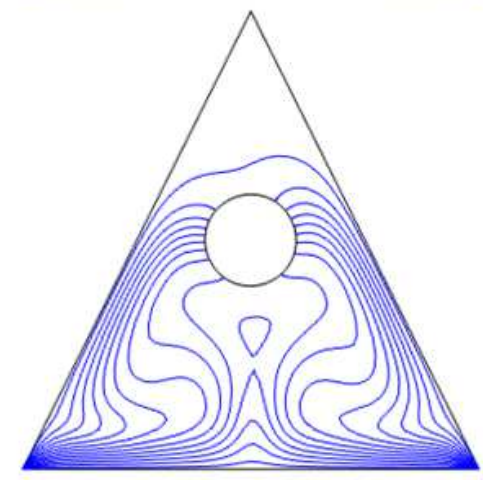

$R=0.01$

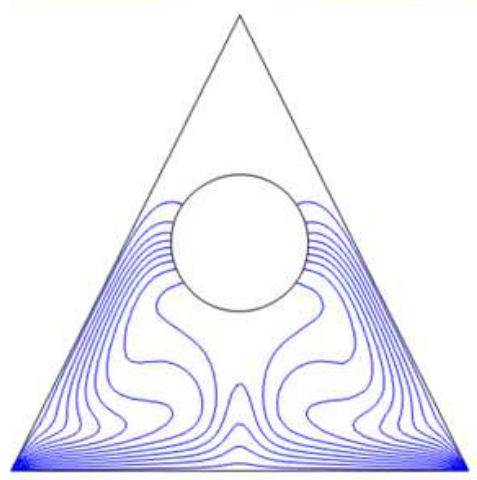

$R=0.015$

Fig. 4. Isotherms for different values of radius of the circular body $R$ and heat generation parameter $\lambda$ with $R a=10^{5}, \operatorname{Pr}=0.71$ and $D a=0.4$. 


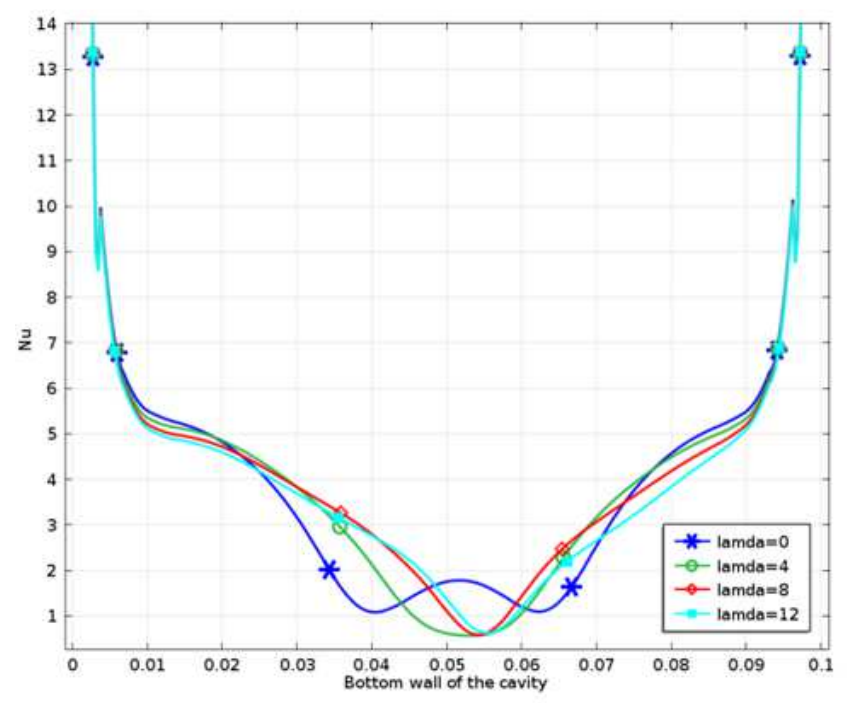

(a)

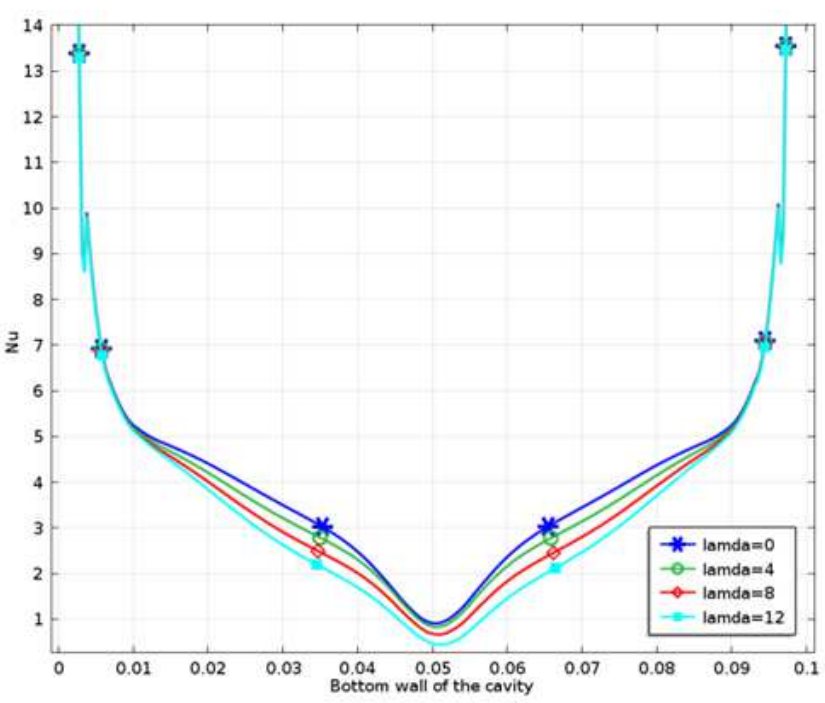

(b)

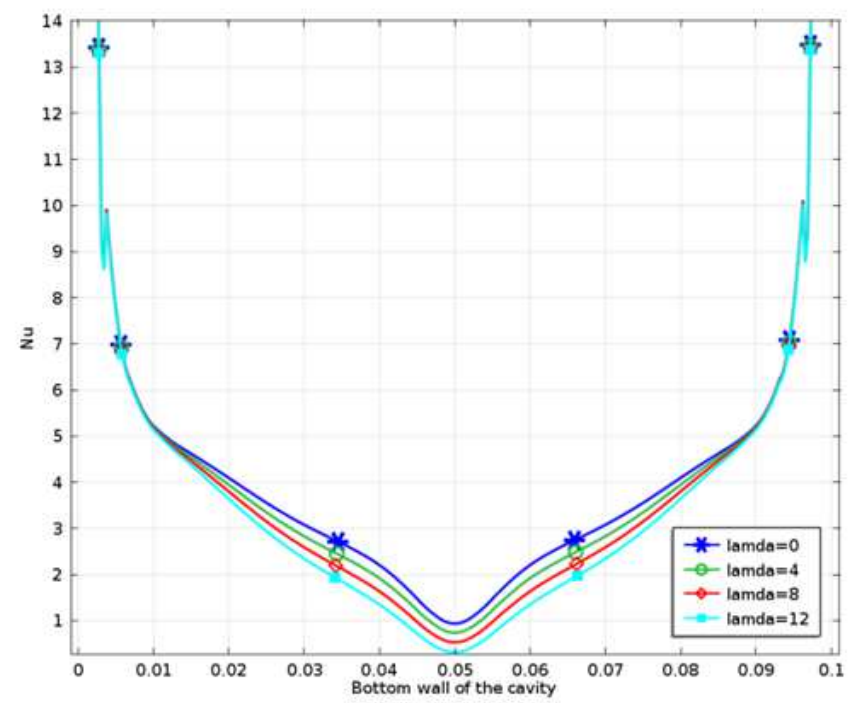

(c)

Fig. 5. The variation of average Nusselt number $N u$ along the hot bottom wall for different heat generation parameter $\lambda$ at (a) $R=0.005$, (b) $R=0.01$ and (c) $R=0.015$.

Fig. 3-4 represents the effects of the raduis of the circular body with the four different values of heat generation parameter $\lambda$ on streamlines as well as isotherms while $\operatorname{Pr}=0.71, R a=10^{5}$ and $D a=0.4$. For $\quad \lambda=0$ and $R=$ 0.005 , two vortices are formed within the cavity where the large vortex near the left inclined wall contains the circular obstacle and dominates the other vortex formed near the right bottom corner of the triangular cavity. For increasing value of $\lambda$, the size of the large vortex increases and occupies maximum space of the cavity due to increasing heat generation and size of the secondary vortex decreases. As $\lambda$ increases from 0 to 12 , almost two equal size vortices are generated just below the circular body within the triangular cavity for $R=0.01$ and $R=0.015$. It must be noticed that no significant change in streamlines occurs for increasing heat generation. This happens because of the increasing size of the solid body which gives rise to a decrease in space available for the flow induced by the heat generation. No flow occurs in the top portion of the cavity for increasing heat generation and size of the circle.

Since the bottom surface is heated constantly, heated boundary layer is develops adjacent to the bottom wall which is shown in Fig. 4. Due to the buoyancy effect, the hot fluid inside the boundary layer moves upward from the bottom left tip. Clustered isotherms are shown close to bottom surface in the absence of heat generation. It can be seen that in the presence of heat generation and for increasing value of $\lambda$ the region of clustered isotherms moves toward the cold surfaces of the cavity for all values of $R$. This happens due to high temperature differences between the hot and cold fluid. For $R=0.01$ and $\mathrm{R}=0.015$, symmetrical isotherms are formed.

Fig. 5 shows the variation of average Nusselt number $\mathrm{Nu}$ along the heated wall of the cavity for different heat generation parameter $\lambda$ at different size of the circular body. 
From fig. 5 (a)-(c), it can be observed that $N u$ decreases with the increasing value of heat generation parameter $\lambda$. This is expected as the heat generation process will increase the fluid temperature near the bottom surface, resulting in increased resistance to the transfer of heat in vertical direction. It is also noticed that $\mathrm{Nu}$ decreases with the increasing value of $R$ as large circle reduce the flow surface.

\section{Conclusion}

A numerical study is performed to investigate the flow and temperature field when a circular body is inserted in a triangular porous enclosure in presence of heat generation. The outcomes of the existing analysis are as follows:

- The fluid flow and temperature field strongly depend on

\section{Nomenclature}

$C_{p} \quad$ specific heat at constant pressure $\left[\mathrm{Jkg}^{-1} \mathrm{~K}^{-1}\right]$

$\mathrm{Da} \quad$ Darcy parameter

$g$ gravitational acceleration $\left[\mathrm{ms}^{-2}\right]$

$k$ thermal conductivity of the media $\left[\mathrm{Wm}^{-1} \mathrm{~K}^{-1}\right]$

$K \quad$ permeability of the porous media $\left[\mathrm{m}^{2}\right]$

$l \quad$ length of the inclined wall $[\mathrm{m}]$

$r \quad$ radius of the circular body [m]

$R \quad$ Dimensionless radius of the circle

$L \quad$ height of the triangle [m]

$\mathrm{Nu} \quad$ Nusselt number

$p \quad$ fluid pressure $[\mathrm{Pa}]$

$\mathrm{Pr} \quad$ Prandtl number

$\mathrm{Ra}$ Rayleigh number

$T$ temperature $\left[{ }^{\circ} \mathrm{C}\right]$

$u, v \quad$ velocity in $x, y$ direction $\left[\mathrm{ms}^{-1}\right]$

$U, V$ dimensionless velocity components

$U_{0} \quad$ reference velocity, $\alpha / L$

$x, y \quad$ Cartesian coordinates [m]

$X, Y \quad$ dimensionless coordinates

\section{References}

[1] D. A. Nield, A. Bejan, Convection in Porous Media, second ed., Springer, NY, 2006.

[2] D. B. Ingham, I. Pop, Transport Phenomena in Porous Media II, Pergamon, 2005.

[3] S. Ostrach, Natural convection in enclosures, Adv. Heat Transfer 8 (1972) 161-227.

[4] M. A. Hossain, M. Wilson, Natural convection flow in a fluidsaturated porous medium enclosed by non-isothermal walls with heat generation, Int. J. Therm. Sci. 41 (2002) 447-454.

[5] S. Parvin and R. Nasrin, Analysis of the Flow and Heat Transfer Characteristics for MHD Free Convection in an Enclosure with a Heated Obstacle, Nonlinear Analysis: Modelling and Control, 16 (2011) 89-99.

[6] H. Asan, L. Namli, Laminar natural convection in a pitched roof of triangular cross-section: Summer day boundary the presence of the circular body obstructs the flow and temperature fields.

- The heat generation effect is negligible for large size of circular body.

- The average Nusselt number significantly worse with increasing both heat generation and size of the circle.

\section{Acknowledgements}

This work is supported by the Department of Mathematics, Bangladesh University of Engineering and Technology and Department of Natural Science, Stamford University Bangladesh.

\section{Greek symbols}

$\alpha \quad$ thermal diffusivity $\left[\mathrm{m}^{2} \mathrm{~s}^{-1}\right]$

$\beta \quad$ thermal expansion coefficient of fluid $\left[\mathrm{K}^{-1}\right]$

$\theta$ dimensionless temperature

$\gamma \quad$ fluid ratio of specific heat

$\lambda$ dimensionless heat generation parameter

$\mu \quad$ dynamic viscosity $\left[\mathrm{Pas}^{-1}\right]$

$v \quad$ kinematic viscosity, $\mu / \rho\left[\mathrm{m}^{2} \mathrm{~s}^{-1}\right]$

$\rho \quad$ fluid density $\left[\mathrm{kgm}^{-3}\right]$

$\varepsilon \quad$ porosity

\section{Subscript \\ c cold \\ $h$ hot}

[7] V. A. Akinsete, T. A. Coleman, Heat transfer by steady laminar free convection in triangular enclosures, Int. J. Heat Mass Transfer 25 (1982) 991-998.

[8] Y. Varol, H. F. Oztop, A. Varol, Free convection in porous media filled right-angle triangular enclosures, Int. Comm. Heat Mass Transfer 33 (2006) 1190-1197.

[9] A. C. Baytas, I. Pop, Free convection in oblique enclosures filled with a porous medium, Int. J. Heat Mass Transfer 42 (1999) 1047-1057.

[10] Y. Varol, H. F. Oztop, Free convection in a shallow wavy enclosure, Int. Comm. Heat Mass Transfer 33 (2006) 764-771.

[11] Y. Varol, A. Koca, H. F. Oztop, Numerical convection in a triangle enclosure with flush mounted heater on the wall, Int. Comm. Heat Mass Transfer 33 (2006) 951-958.

[12] E. Ridouane, A Campo, M. Hasnaoui, Benefits derivable from connecting the bottom and top walls of attic enclosure with insulated vertical side walls, Numer. Heat Transfer Part AApplications 49 (2006) 175-193. 
[13] K. A. Joudi, I. A. Hussein, A. A. Farhan, Computational model for a prism shaped storage solar collector with a right triangular cross section, Energy Conservation and Management 45 (2004) 391-409.

[14] S. C. Tzeng, J. H. Liou, R. Y. Jou, Numerical simulation-aided parametric analysis of natural convection in a roof of triangular enclosures, Heat Transfer Engrg. 26 (2005) 69-79.

[15] A. Bejan, D. Poulikakos, Natural convection in an atticshaped space filled with porous material, J. Heat Transfer 104 (1982) 241-247.

[16] Y. Varol, H. F. Oztop, A. Varol, Free convection in porous media filled right-angle triangular enclosures, Int. Comm. Heat Mass Transfer 33 (2006) 1190-1197.

[17] T. Basak, C. Thirumalesha, S. Roy, Finite element simulations of natural convection in a right-angle triangular enclosure filled with a porous medium: effects of various thermal boundary conditions, J. Porous Media 11 (2008) 159-78.
[18] Y. Varol, H.F.Oztop, T. Yilmaz, Two dimensional natural convection in a porous triangular enclosure with a square body, Int. Commun.Heat Mass Transfer 34 (2007) 238-247.

[19] A. Amine, J.K. Platten, M. Hasnaoui, Thermal convection around obstacles: the case of Sierpinski carpets, Exp. Fluids 36 (2004) 717-727.

[20] M.Y. Ha, I.K. Kim, H.S. Yoon, K.S. Yoon, J.R. Lee, S. Balachandar, H.H. Chun, Two-dimensional and unsteady natural convection in a horizontal enclosure with a square body, Numer. Heat Transf., A Appl. 41 (2002) 183-210.

[21] H.F. Oztop, I. Dagtekin, M. Duranay, Analysis of natural convection problem in a cavity with partially heated and block inserted, Proceedings of 13rd Heat Science and Technology Congress, Turkey, 2001, pp. 217-222, (In Turkish).

[22] Suvash C. Saha, Y. T. Gu, Free convection flow in a triangular enclosure with fluid-saturated porous medium enclosed with heat generation, J. ANZIAM 53 (2012) C127-C141. 